\title{
The psychological impact on patients with memory disorders and their caregivers during COVID-19
}

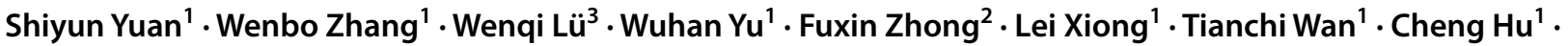 \\ Wenkai Yang ${ }^{1} \cdot$ Chenxi Chen $^{1}$ - Daijing Luo ${ }^{3} \cdot{\text { Binjie } \operatorname{Tan}^{3} \cdot \text { Chengcheng Huang }}^{1} \cdot$ Weihua Yu ${ }^{2} \cdot$ Yang Lü ${ }^{1} \mathbb{D}$
}

Received: 18 January 2021 / Accepted: 9 June 2021 / Published online: 23 June 2021

( $)$ The Author(s), under exclusive licence to Springer Nature Switzerland AG 2021

\begin{abstract}
Background COVID-19 is erupting globally. Mass quarantine had been implemented all around China which could influence the psychological status of patients with memory disorders and their caregivers.

Aim To investigate the psychological impact of mass quarantine on patients with memory disorders and their caregivers in China.

Methods We completed a cross-sectional study in 787 patients and their caregivers registered from 2010 to 2019 in Memory Clinic, The First Affiliated Hospital of Chongqing Medical University, by telephone interviews. The performance in neuropsychiatric symptoms (NPSs), sleep, nutrition, chronic diseases of patients, and the burden of care, anxiety and depression of caregivers was assessed by six assessment scales (MNA-SF, PSQI, NPI, RSS, PHQ-9 and GAD-7).

Results Only 68 (8.6\%) patients worried about the outbreak of COVID-19. The prevalence of NPSs among all subjects was nearly $60.0 \%$. Approximately $50.0 \%$ of the caregivers reported distress. More than $70.0 \%$ of patients remained stable in NPSs. However, anxiety, depression, aberrant motor disorder and delusion were exacerbated $(22.9 \%, 18.6 \%, 17.1 \%$ and $16.8 \%$, respectively). Appetite and eating disorder led alleviation rate by $25.8 \%$ while disappearing rate of agitation led by 5.8\%. $7.5 \%$ of caregivers manifested depressive symptoms while $4.9 \%$ expressed anxiety symptoms, and $40.8 \%$ showed care burden. The coefficients of RSS and PHQ-9, RSS and GAD-7, RSS and NPI-D, PHQ-9 and GAD-7 were 0.7, 0.5, 0.5 and 0.6 , respectively $(p<0.01)$.
\end{abstract}

Conclusion Changes in NPSs during COVID-19 were observed in some patients with memory disorders and their caregivers, and adherence to medication contributed to the stabilization of NPSs.

Keywords COVID-19 $\cdot$ Psychological impact $\cdot$ Memory disorders $\cdot$ The elderly $\cdot$ Caregiver

Shiyun Yuan, Wenbo Zhang, Wenqi Lü, Wuhan Yu, Fuxin Zhong contributed equally to this study.

Weihua Yu

yuweihua@cqmu.edu.cn

Yang Lü

yanglyu@ hospital.cqmu.edu.cn

1 Department of Geriatrics, The First Affiliated Hospital of Chongqing Medical University, No.1 Youyi Road, Yuzhong District, Chongqing 400016, China

2 Institutes of Neuroscience, Chongqing Medical University, No.1 Yixueyuan Road, Yuzhong District, Chongqing 400016, China

3 Chongqing Medical University, Chongqing, China

\section{Introduction}

Coronavirus disease 2019 (COVID-19) has become a globally spread disease. Mass quarantine had been implemented all around China after the lockdown of Wuhan on January 23, $2020[1,2]$. It was estimated that about 760 million people were confined to their homes [3, 4]. Government of Chongqing initiated a first-level response on January 24, 2020 to contain the spread of COVID-19. Since then, draconian home quarantine had been strictly enforced throughout all communities and villages of Chongqing to limit population mobility [5-7].

In general, quarantine contributed to psychological impacts on involved people, such as fear, depression, stress, low mood, irritability, insomnia, post-traumatic stress symptoms and anger. However, positive response was also 
reported even though it was rare $[8,9]$. As the elderly with memory disorders were considered to be vulnerable and susceptible to both COVID-19 and its stress, they should be better taken care of since decreased perception of the surrounding and the accompanied neuropsychiatric symptoms (NPSs) might be manifested at any occasion [10, 11]. However, based on the fact that it was the first time for Chongqing even for China to implement mass quarantine and sudden changes were immediately occurred to all citizens, the impact on the elderly with cognitive impairment during this particular period was unknown.

Previous studies were mainly focused on prevention, diagnosis and treatment of COVID-19 and situation of medical personnel but given the fact that the majority of population is still or will be under quarantine [3], understanding their situation, especially the elderly with memory disorders, shares the same importance. Although several reports have reported on the effects of COVID-19 pandemic confinement in patients with cognitive impairment in other countries, such as Spain [12,13] and Italy [14-17], the psychological impact on patients with memory disorders and their caregivers during COVID-19 in China has not yet been reported. Therefore, our team conducted a telephone interview on all registered patients in our Memory Clinic, focusing on psychological-related performances of the elderly with memory disorders by evaluating the changes in lifestyle, nutritional status, sleep and NPSs during quarantine.

We hope that our study could provide useful information and references to other provinces of China as well as countries worldwide for seeking better management of patients with memory disorders at this particular time when the whole world should act as one.

\section{Materials and methods}

\section{Procedures}

This cross-sectional study was conducted during February 11 to 23,2020. The investigation was carried out by 15 investigators with professional training and interview experience. Our study consisted of five parts: (1) demographic information; (2) exposure history of COVID-19; (3) medication for dementia and other chronic diseases; (4) status of nutrition, sleep and neuropsychiatric symptoms (NPSs) of patients; and (5) the burden of care, anxiety, depression of caregivers. All the interviews were carried out over the telephone, followed by quality control and statistical analysis.

\section{Subjects}

A total of 1,908 patients from Memory Clinic of Department of Geriatrics, The First Affiliated Hospital of Chongqing
Medical University from 2010 to 2019 were included in this study, which was approved by the Ethics Committee of The First Affiliated Hospital of Chongqing Medical University (Certificate No.20200301).

\section{Public activities of Memory Clinic during the COVID-19 outbreak}

Our Memory Clinic is located at the junction of Yuzhong, Jiulongpo and Nan'an Districts, Chongqing. As a routine, the patients and caregivers were invited to join Tencent social platform (QQ) for online consultation. During the COVID19 outbreak, we helped patients and caregivers in consulting mental and behavioral problems, managing chronic diseases, buying drugs online and mailing drugs from hospital. All the activities were cost-free.

\section{Instruments}

During home isolation, only the scales suitable for telephone interview were selected in our study, which have been widely used in a large number of reported studies previously [12-14] and were targeted to the filed that we were interested in. Six assessment scales were applied in our study, including Mini-Nutritional Assessment Short Form (MNA-SF), Pittsburgh Sleep Quality Index (PSQI), Neuropsychiatric inventory (NPI), Relative stress scale (RSS), Patient Health Questionnaire-9 (PHQ-9) and Generalized Anxiety Disorder scale (GAD-7) (see supplementary for rating). The change of each NPS was also provided by caregivers, shifting into new-onset, exacerbated, stable, alleviated, and disappeared.

\section{Data analysis}

Continuous measurements were presented as mean (SD) if they are normally distributed or median (IQR) if not, and categorial variables as count (\%). All statistical analyses were performed by SPSS 26.0 (IBM, Armonk, NY). Demographic characteristics were evaluated by frequency distributions. ANOVA and chi-square analyses were performed to compare groups on quantitative continuous and on categorical variables, respectively. The influence of medication on changes of NPSs among patients with mild cognitive impairment (MCI), Alzheimer's disease (AD), non-AD dementia and others was compared by Mann-Whitney Test. The effects of appetite and body weight on nutritional status were analyzed by Spearman's rank correlation analysis and Kruskal-Wallis test, respectively. Spearman's rank correlation coefficient analysis was performed to analyze the correlations between every two of RSS, caregiver distress (NPI-D), PHQ-9 and GAD-7. 


\section{Results}

\section{Demographics}

787 registered patients completed our survey. The main diagnoses among respondents in this study were presented in Fig. 1a with AD taking up the biggest proportion. Subjects were then regrouped by diagnosed as $\mathrm{MCI}, \mathrm{AD}$, non- $\mathrm{AD}$ dementia and other diseases (Table 1). The patients were 275 male and 512 females with an average of age 74.5 years old. Although female took up nearly $64.9 \%$ of the total subjects, the distribution of sex in each group showed no statistic difference $(F=3.3, p=0.345)$. The respondents came from 11 provinces and 1 municipality of China, where Chongqing took up $90.3 \%$ of the enrolled, followed by Sichuan province with $4.8 \%$ (Online Resource 1). a

Frequency of Each Diagnosis

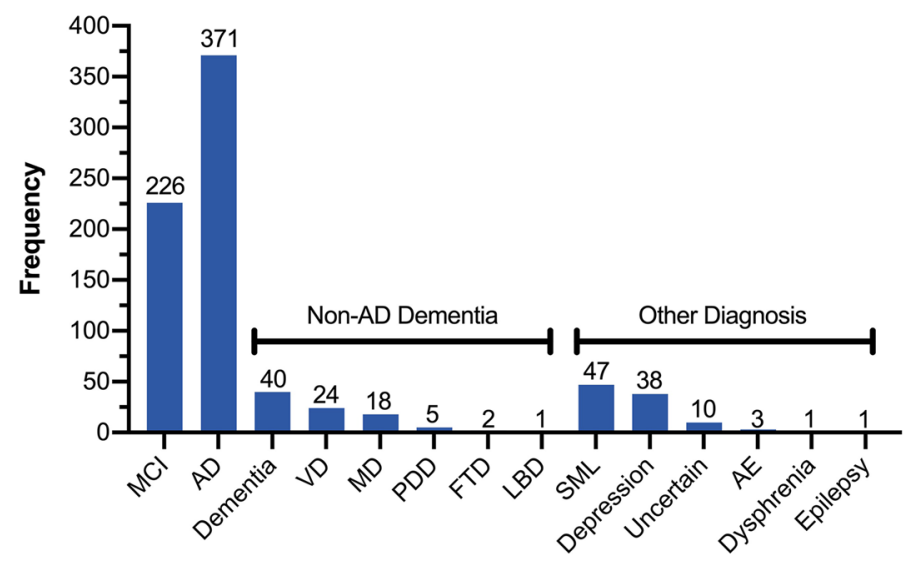

b

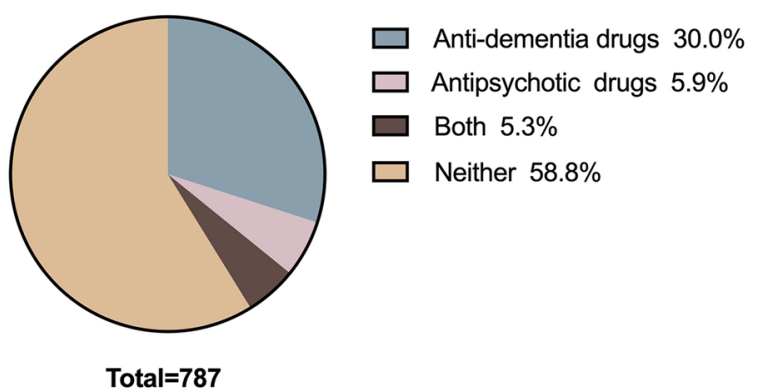

Fig. 1 Diagnosis among respondents in Memory Clinic and percentage of drug usage in all respondents. a The majority of patients had AD (371, 47.1\%), followed by MCI $(226,28.7 \%)$ and SML (47, 5.9\%). $A D$ Alzheimer's disease, MCI Mild cognitive impairment, $S M L$ Subjective memory loss, VD Vascular dementia, PDD Parkinson's disease dementia, FTD Frontotemporal dementia. b Of all
787 subjects, nearly $30.0 \%$ were taking anti-dementia drugs, and an equally around $5.0 \%$ of patients were using antipsychotics or both, remaining 58.8\% taking neither. Both $=$ Both antipsychotics and antidementia drugs were used. Neither $=$ Neither antipsychotics nor antidementia drugs were used

Table 1 Demographic and general evaluative outcomes of participants enrolled

\begin{tabular}{|c|c|c|c|c|c|c|c|c|c|c|c|c|}
\hline & \multicolumn{2}{|c|}{ Total $n=787$} & \multicolumn{2}{|c|}{ MCI $n=226$} & \multicolumn{2}{|c|}{$\mathrm{AD} n=371$} & \multicolumn{2}{|c|}{$\begin{array}{l}\text { Non-AD Dementia } \\
n=90\end{array}$} & \multicolumn{2}{|c|}{$\begin{array}{l}\text { Other Diagnosis } \\
n=100\end{array}$} & \multirow[t]{2}{*}{$\begin{array}{l}\chi^{2} \\
\text { or } F\end{array}$} & \multirow[t]{2}{*}{$p$} \\
\hline & $N$ & $\%$ & $N$ & $\%$ & $N$ & $\%$ & $N$ & $\%$ & $N$ & $\%$ & & \\
\hline Male & 275 & 34.9 & 75 & 33.2 & 131 & 35.3 & 36 & 40.0 & 33 & 33.0 & 3.3 & .345 \\
\hline \multirow[t]{2}{*}{ Female } & 512 & 65.1 & 151 & 66.8 & 240 & 64.7 & 54 & 60.0 & 67 & 67.0 & & \\
\hline & Mean & SD & Mean & SD & Mean & SD & Mean & SD & Mean & SD & & \\
\hline \multirow[t]{2}{*}{ Age } & 74.5 & 9.9 & 72.3 & 9.7 & 77.3 & 8.8 & 77,0 & 8.8 & 67.1 & 9.6 & 39.7 & $<.001$ \\
\hline & Median & IQR & Median & IQR & Median & IQR & Median & IQR & Median & IQR & & \\
\hline NPI & 2 & $0-9$ & 0 & $0-4$ & 4 & $0-12$ & 4 & $0-10.2$ & 0.5 & $0-7.5$ & 35.7 & $<.001$ \\
\hline Caregiver Distress & 0 & $0-4$ & 0 & $0-2$ & 2 & $0-6$ & 2 & $0-6$ & 0 & $0-2$ & 46.5 & $<.001$ \\
\hline MNA-SF* & 11 & $10-12$ & 12 & $11-13$ & 11 & $9-12$ & 11 & $10-12$ & 13 & $11-14$ & 68.1 & $<.001$ \\
\hline PSQI* & 4 & $2-7$ & 4 & $2-7$ & 4 & $2-7$ & 4 & $2-8$ & 5 & $2-8$ & 29.5 & .532 \\
\hline RSS* & 0.5 & $0-7$ & 0 & $0-2$ & 3 & $0-10$ & 3 & $0-10.5$ & 0 & $0-1$ & 89.7 & $<.001$ \\
\hline PHQ-9* & 0 & $0-1$ & 0 & $0-0$ & 0 & $0-2$ & 0 & $0-1.25$ & 0 & $0-0$ & 24.8 & $<.001$ \\
\hline GAD-7* & 0 & $0-0$ & 0 & $0-0$ & 0 & $0-1$ & 0 & $0-1$ & 0 & $0-0$ & 19.0 & $<.001$ \\
\hline
\end{tabular}

*MNA-SF: 760 cases enrolled and 26 cases excluded due to incomplete data

PSQI: 751 cases enrolled and 36 cases excluded due to incomplete data

RSS \& PHQ-9 \& GAD-7: 737 cases enrolled and 50 cases excluded due to living alone 


\section{Exposure history to COVID-19}

In our study, only 4 patients had history of contact with people from Wuhan or Hubei, and 3 had traveled there. Neither the subjects nor their families were diagnosed or suspected with COVID-19. There were only 68 (8.6\%) patients worried about the outbreak of COVID-19, most of whom showed anxiety and nervousness (83.8\%) (Table 2).

\section{Medication and chronic disease management}

Among all respondents, nearly $30.0 \%$ of patients were taking anti-dementia drugs while only $5.9 \%$ of them were using antipsychotics with another 5.3\% taking both (Fig. 1b). Except for dementia, $49.7 \%$ of patients had other chronic diseases with a maximum of 5 . During the outbreak of COVID-19, $93.4 \%$ of patients with chronic diseases were stable. Among patients with chronic disease instability, $38.5 \%$ did not take medication regularly.

Table 2 Specific symptoms of anxiety due to COVID-19 outbreak

\begin{tabular}{lrr}
\hline Specific symptoms of anxiety about the outbreak & N & $\%$ \\
\hline Chest tightness, palpitations and dyspnea & 5 & 7.4 \\
Feeling upset, worried and irritable & 57 & 83.8 \\
Cannot stop or control worry & 13 & 19.1 \\
Very nervous and hard to relax & 20 & 29.4 \\
Easily upset or irritated & 17 & 25.0 \\
\hline
\end{tabular}

There were 68 patients worried about the outbreak of COVID-19, 57 of them stated the feeling of upset, worried and irritable due to COVID-19 outbreak, taking up $83.8 \%$, followed by near $30.0 \%$ of being very nervous and having difficulty to relax. Only 5 respondents claimed the chest tightness, palpitations and dyspnea

\section{Evaluation for nutrient status and sleep quality}

761 cases enrolled with full data and 26 cases excluded due to the lack of data on weight. Only $3.3 \%$ of the elderly suffered from malnutrition, $51.4 \%$ and $45.3 \%$ were at risk of malnutrition and well-nourished, respectively. $7.8 \%$ of the participants stated loss of appetite, whereas $2.1 \%$ showed an obvious increase in appetite. $4.1 \%$ of participants lost weight while $6.0 \%$ gained (Table 3 ). In terms of sleep quality, 479 were rated as "good" and 286 as "poor".

\section{The prevalence, stability of NPSs and the influence of medication}

The prevalence of NPSs (NPI $\geq 1$ ) among all subjects was $57.9 \%$. Among patients with subjective memory loss (SML), depression, MCI, AD and Non-AD dementia, the prevalence of NPSs (NPI $\geq 1$ ) was 27.7, 73.7, 43.4, $67.1 \% 65.6 \%$, respectively. Patients with depression showed highest prevalence as well as mean score, followed by AD subjects, while participants with SML displayed with the lowest prevalence of NPS and mean score of NPI $(p<0.001)$ (Online Resource $2 \mathrm{a}, \mathrm{b})$. In patients with depression, depression showed the highest prevalence $(57.9 \%)$, followed by sleep disturbance (36.8\%) and anxiety (31.6\%) (Online Resource $2 \mathrm{~d}$ ). While anxiety and sleep disturbance showed the highest prevalence (both $10.6 \%$ ), followed by depression (8.5\%) in patients with SML (Online Resource $2 \mathrm{c}$ ). The high prevalence of depression, anxiety and sleep disturbance in SML and depression groups indicated that these domains might be affected by COVID-19 outbreak. Correspondingly, approximately $50.0 \%$ of the caregivers reported distress
Table 3 Appetite and weight change of the study population according to categories of nutritional status

\begin{tabular}{|c|c|c|c|c|c|c|c|c|c|}
\hline \multirow[t]{3}{*}{ Parameters } & \multirow{2}{*}{\multicolumn{2}{|c|}{ Total }} & \multicolumn{6}{|c|}{ Nutritional status } & \multirow[t]{3}{*}{$P$} \\
\hline & & & \multicolumn{2}{|c|}{$\begin{array}{l}\text { Malnutrition } \\
n=25\end{array}$} & \multicolumn{2}{|c|}{$\begin{array}{l}\text { At risk of malnu- } \\
\text { trition } n=391\end{array}$} & \multicolumn{2}{|c|}{$\begin{array}{l}\text { Well-nourished } \\
n=345\end{array}$} & \\
\hline & $N$ & $\%$ & $N$ & $\%$ & $N$ & $\%$ & $N$ & $\%$ & \\
\hline \multicolumn{10}{|l|}{ Food intake } \\
\hline Stable & 686 & 90.1 & 11 & 44.0 & 346 & 88.5 & 329 & 95.4 & $<0.001$ \\
\hline Decreased & 59 & 7.8 & 13 & 52.0 & 40 & 10.2 & 6 & 1.7 & \\
\hline Increased & 16 & 2.1 & 1 & 4.0 & 5 & 1.3 & 10 & 2.9 & \\
\hline \multicolumn{10}{|c|}{ Weight change } \\
\hline Stable & 593 & 77.9 & 5 & 20.0 & 276 & 70.6 & 312 & 90.4 & $<0.001$ \\
\hline Lost & 31 & 4.1 & 8 & 32.0 & 22 & 5.6 & 1 & 0.3 & \\
\hline Gained & 46 & 6.0 & 0 & 0.0 & 14 & 3.6 & 32 & 9.3 & \\
\hline Unknown & 91 & 12.0 & 12 & 48.0 & 79 & 20.2 & 0 & 0.0 & \\
\hline
\end{tabular}

761 cases enrolled with full data and 26 cases excluded due to the lack of data on weight. $7.8 \%$ of the participants stated loss of appetite, whereas $2.1 \%$ showed an obvious increase in appetite. Compared with other two categories, malnutrition displayed highest rate of decreased food intake by $52.0 \%$, and correspondingly, took up $32.0 \%$ of weight loss 
Fig. 2 The prevalence of NPSs among participants in each group. NPSs neuropsychiatric symptoms, NPI Neuropsychiatric inventory, $A D$ Alzheimer's disease, $M C I$ Mild cognitive impairment, $A M D$ aberrant motor disorder, $A E D$ appetite and eating disorder. Overall, nearly $60.0 \%$ of subjects had NPSs (NPI $\geq 1$ ) with a correspondent of around $50.0 \%$ of caregiver distress. Sleep disturbance, apathy, irritability and delusion were more prominent during quarantine with prevalence of more than $20.0 \%$. In addition, $\mathrm{AD}$ patients took up the majority proportion in each NPS

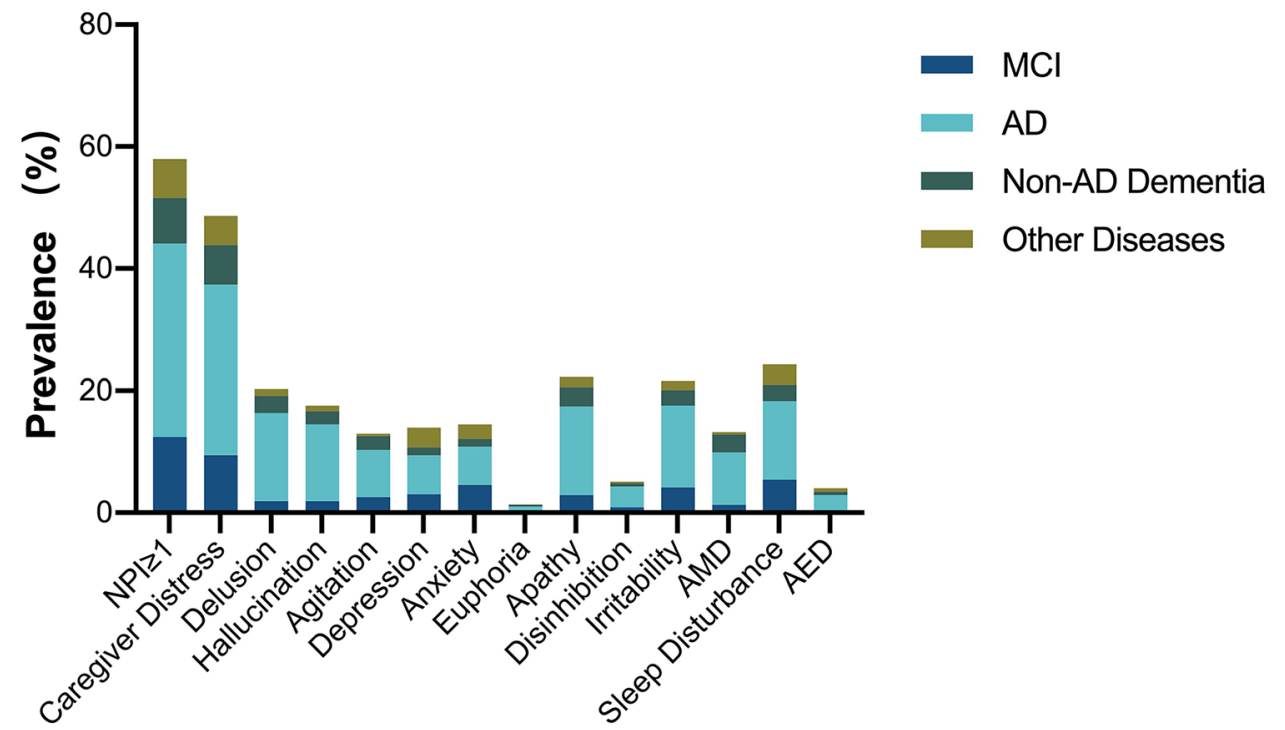

(NPI-D $\geq 1$ ) (Fig. 2). Within the 12 domains, the prevalence rates of sleep disturbance, apathy, irritability and delusion were more than $20.0 \%$, while hallucination, agitation, depression and anxiety were above $15.0 \%$.

During this quarantine, more than $70.0 \%$ of patients with NPSs remained relatively stable. The overall status was analyzed and displayed in Fig. 3. There were 89 newonset cases, taking up $11.0 \%$ of the whole subjects, while anxiety, depression, aberrant motor disorder (AMD) and delusion were prominently exacerbated (22.9, 18.6, 17.1 and $16.8 \%$, respectively). Surprisingly, appetite and eating disorder (AED) showed substantial alleviation by $25.8 \%$ while agitation led the highest disappearing change by $5.8 \%$.

Adherence to medication contributed to the stabilization of NPSs. It was found that patients who followed the prescription conveyed higher tendency to perform better in agitation, depression, anxiety, apathy and irritability (all $P<0.05)$ (Online Resource 3 ).

\section{Burden of care for caregivers}

737 subjects provided useful data while 50 patients lived alone. $96.6 \%$ of caregivers were family members, such as children or spouses, and $3.4 \%$ were employed carers. More than half of the caregivers reported no care burden ( RSS $=0$ ), while $49.8 \%$ stated different levels of care burden with an average RSS of 8.6, among whom $33.2 \%$ were worried about the patient's accident, $34.5 \%$ were in need of a break, and $29.1 \%$ could no longer cope with this situation, indicating a high degree of distress was endured by caregivers during this quarantine (Online Resource 4).

\section{Anxiety and depression of caregivers}

In our survey, 737 caregivers finished PHQ-9 and GAD-7. More than $90.0 \%$ individuals showed no depressive emotions while $7.5 \%$ did, among whom the degrees of depression were centralized in mild $(6.4 \%)$ and moderate $(1.1 \%)$ with no moderately severe or severe cases. Anxiety symptoms were shown in only 36 caregivers, taking up $4.9 \%$ of the whole, and most of whom were at mild degree $(83.3 \%)$ (Table 4).

\section{Associations among burden of care, anxiety and depression of caregivers}

The coefficients of RSS and PHQ-9, RSS and GAD-7, RSS and NPI-D, PHQ-9 and GAD-7 were 0.7, 0.5, 0.5 and 0.6, respectively $(p<0.01)$ (Online Resource 5), meaning the patient's NPSs was associated with caregiver's burden, anxiety and depression.

Further, the correlation of NPI-D, PHQ-9 and GAD-7 with three RSS domains were analyzed in Online Resource 6 , showing that caregivers' depression was highly correlated with personal distress $(\rho=0.5)$ and domestic upset $(\rho=0.7)$, while the negative feelings for caregivers generated mostly from the NPSs of patients $(\rho=0.5)$.

\section{Discussion}

Previous researches on psychological status were mainly focused on patients with contagious disease, close contacts and medical staff [18]. However, there is still a need for relevant researches on psychological status of the elderly 

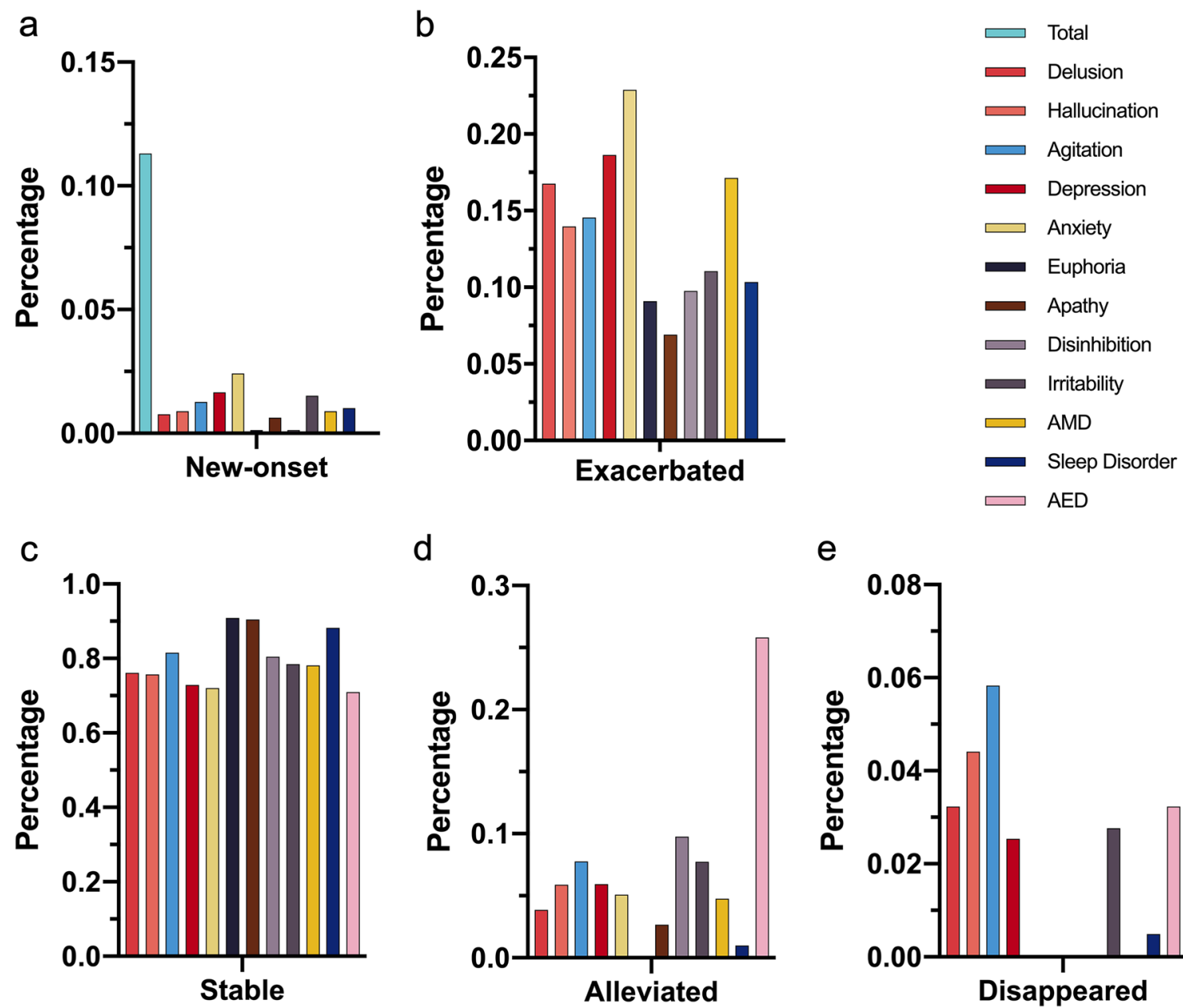

d

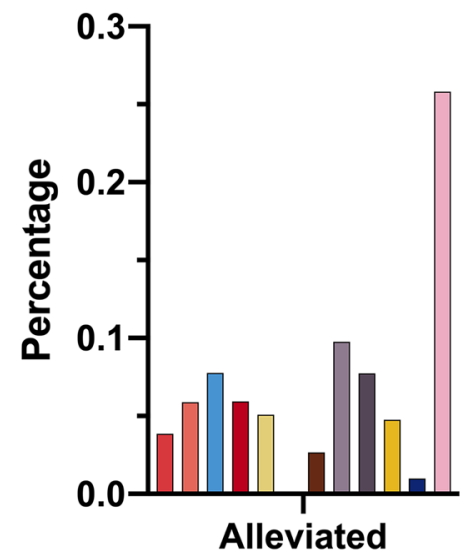

e

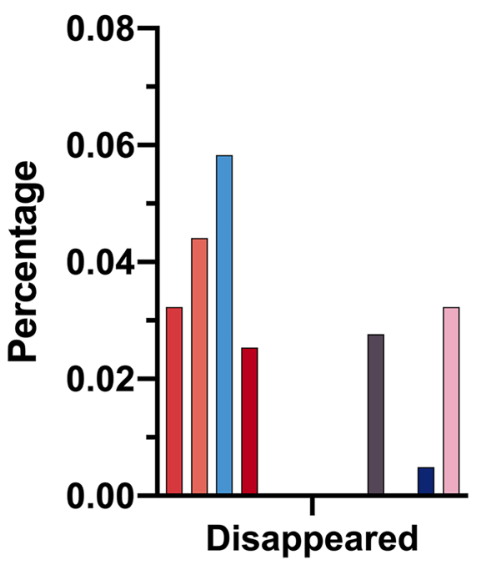

Fig. 3 Rate of changes for each NPS during COVID-19 outbreak and quarantine at home. a Rates for the total and each new-onset NPSs, where in general, $11.0 \%$ NPSs onset during quarantine, and anxiety ranked first. b Exacerbated rate of each NPSs, where, again, anxiety led the rate, followed by depression, AMD and delusion. c Rates of stabled NPSs, where more than $70.0 \%$ of patients remained stable. d Rates of alleviated NPSs, where AED showed the leading rate of

Table 4 The distribution of depression and anxiety in caregivers

\begin{tabular}{lrrlll}
\hline Classification & \multicolumn{2}{c}{ Depression } & & \multicolumn{2}{c}{ Anxiety } \\
\cline { 2 - 3 } & $N$ & & & $N$ & $\%$ \\
\hline None & 682 & 92.5 & & 701 & 95.1 \\
Mild & 47 & 6.4 & & 30 & 4.1 \\
Moderate & 8 & 1.1 & & 5 & 0.7 \\
Moderately severe & 0 & 0.0 & & N/A & N/A \\
Severe & 0 & 0.0 & & 1 & 0.1 \\
\hline
\end{tabular}

737 cases enrolled and 50 cases excluded due to living alone. More than $90.0 \%$ individuals showed no depressive emotions. Among whom the degrees of depression were centralized in mild (6.4\%) and moderate $(1.1 \%)$ with no moderately severe or severe cases. Anxiety symptoms were shown in only 36 caregivers, taking up $4.9 \%$ of the whole, and most of whom were at mild degree $(83.3 \%)$
$25.8 \%$, followed by disinhibition, irritability and agitation with lower than $10.0 \%$. e Rates of disappearing NPSs, where agitation led the rate by $5.8 \%$, followed by hallucination with $4.4 \%$. Patients with symptoms like anxiety, euphoria, apathy, disinhibition and AMD showed no change of disappearing. NPSs neuropsychiatric symptoms. $A M D$ aberrant motor disorder. $A E D$ appetite and eating disorder

patients with memory disorders and their caregivers during COVID-19 outbreak especially in China.

In our study, 787 enrolled patients were from 11 provinces of China with an average age of 74.5 years old in which AD took up the biggest proportion. Therefore, the results of our study could partially reflect the mental status of the elderly patients with memory disorders in China during COVID-19.

Respondents of this study do not include people who diagnosed or suspected with COVID-19. Only $8.6 \%$ patients felt anxious and nervousness about the outbreak of COVID-19. This could be attributed to their apathy or decline in cognition to fail to sense and understand the panic-inducing situation of COVID-19 pandemic. 
Our results show that the vast majority of respondents have no significant change in chronic disease control, sleep and nutrition. And mental status is relatively stable during COVID-19 outbreak, which also contributes to the stability of care burden. This is probably because the elderly with cognitive impairment usually show decreased perception of the surroundings; therefore, patients seldom take the initiative to judge and analyze the epidemic situation, which stabilized their mental symptoms. Moreover, adequate life supplies ensured by the government, increased time spent with families and adherence to medication contributed to the stabilization and improvement of NPSs [19-22].

In this study, some NPSs, such as agitation, depression, anxiety, apathy, and irritability, are significantly improved in patients who adhered to anti-dementia and antipsychotic drugs, indicating that receiving treatment in accordance with prescription can enhance the ability to be more optimistic under stress.

Our study found the prevalence of NPSs among patients with depression were higher than the prevalence of all subjects, including sleep disturbance, anxiety, apathy, irritability and delusion. However, participants with SML displayed with the lowest prevalence of NPSs in this study. This may be because patients with SML usually have a normal cognition with self-control compared to those with AD or other types of dementia. And they are often able to maintain a relatively normal life and even work or study ability. Among patients with SML, the three highest prevalence of NPSs were anxiety, depression, and sleep disturbance. Compared with other studies on the psychological reactions of the general Chinese population during COVID-19, the results of our study showed that the prevalence of anxiety, depression, and sleep disturbance in patients with SML was lower [23, 24]. This indicates that the prevalence of NPSs, such as anxiety, depression, and sleep disturbance among patients with SML, we interviewed did not increase due to COVID-19. Of course, the number of SML patients in this study was also small.

However, there is still an average of nearly $15.0 \%$ of NPSs aggravated in patients with memory disorders, of which anxiety and depression were the most exacerbated. Compared with other study [14], the prevalence of depression in our study was lower while anxiety and apathy were higher. This may due to the diverse severity and complexity of COVID-19 outbreaks in different countries and regions, as well as the various countermeasures that were against for. The rest of the aggravated NPSs are mainly psychotic-like symptoms, including delusion, hallucination, agitation, and AMD, that may account for changes in family care arrangements and lifestyle alterations. Because of COVID-19 outbreak, some family members were unable to visit as frequently as before, patients were forced to change to new care environment, and needed additional "running-in" time. Moreover, irregular home life also leads to behavioral problems. During the quarantine, patients' life and normal rest may get irregular due to restrictions on outdoor activities. Besides, increased personal protective measures, such as washing hands frequently and wearing masks, could lead to agitation and irritability because of uncooperative attitude. Previous researches around China have shown the prevalence of depression $(0.5-3.4 \%)$ and anxiety (5.1-5.6\%) in the general population [25-27]. Compared to our results, the proportion of depression in caregivers was higher during the epidemic, while anxiety was consistent, indicating that COVID-19 outbreak had a limited negative impacts on depression and anxiety among caregivers of patients with memory disorders.

The following recommendations are based on our findings and management experience in hopes of being helpful to other countries and regions where COVID-19 outbreaks are ongoing. First, the elderly with memory disorders during the epidemic are preferable to be quarantined at home because they are at high risk for severe cases of COVID19 , and quarantine can help reduce the risk of infection [28]. Second, elderly patients could obtain effective medical consultation and assistance via telephone and internet by physicians during the quarantine [29, 30]. Third, multimode medicine purchase channels should be established due to home quarantine. Fourth, family recreational activities are recommended. It contributes to the maintenance of mental status and sleep routine of the patients [31]. Activities like cognitive games, nostalgic talks, indoor exercises, and housework are quite practicable, like playing mahjong, cards or chess, chatting on old times, reading books for them, listening to music, or doing Tai Chi etc. would be beneficial. Finally, diet and nutrition are important for the elderly to strengthen immunity during the epidemic of contagious disease. Eating several small meals and adoption of balanced diet are good strategy for the elderly with memory disorders [32, 33].

There were also some limitations in our research. The accurate assessment for the severity of cognitive impairment was unable to perform by means of telephone interview. In addition, the sample size was not large enough due to time limitation and single clinic. However, our memory clinic is an epitome of patients with memory disorders and their families in the country; therefore, the present research is representative. In addition, this is a crosssectional study, there are some limitations in revealed psychological impact on patients with memory disorders and their caregivers during COVID-19. To investigate the impact further, a prospective cohort study is under way. 


\section{Conclusion}

In summary, NPSs, such as depression and anxiety, aggravated in some patients with memory disorders and their caregivers during COVID-19, and adherence to medication contributed to the stabilization of NPSs. The epidemic of COVID-19 and its quarantine have brought new challenges to patients with memory disorders and their caregivers, especially in dealing with neuropsychiatric symptoms. During mass quarantine for COVID-19, accompanying time and its quality from caregivers, online medical service, and adherence to medication significantly contributed to the stabilization of the elderly with memory disorders and their care burden. Based on above, we hope that our work can provide scientific reference for improving the management of patients with cognitive impairment in stress events like COVID-19.

Supplementary Information The online version contains supplementary material available at https://doi.org/10.1007/s40520-021-01911-1.

Acknowledgements We thank all respondents and their caregivers involved in the study.

Authors' Contributions YL, WY, SY, WZ, WL, WY, FZ participated in the design of the research scheme. YL, WY, SY, WZ, WL, WY, FZ, LX, TW, CH, WY, DL, BT, CC, CH participated in the telephone survey and data collection. SY was responsible for the aggregation and collation of data. WZ, WL, WY, FZ were responsible for statistics. WZ was responsible for the production of figures. SY, WZ, WL, WY, FZ drafted the manuscript. YL and WY revised the final manuscript. All authors reviewed, revised, and approved the final manuscript.

\section{Funding None.}

\section{Declarations}

Conflict of interest The authors declare that they have no conflict of interest.

Ethical approval The study and all research protocols were approved by the Ethics Committee of The First Affiliated Hospital of Chongqing Medical University.

Human and animal rights This study only included human participants.

Informed consent Informed oral consent was obtained from all participants prior the interviewers.

\section{References}

1. Chen N, Zhou M, Dong X et al (2020) Epidemiological and clinical characteristics of 99 cases of 2019 novel coronavirus pneumonia in Wuhan, China: a descriptive study. Lancet 395:507-513. https://doi.org/10.1016/S0140-6736(20)30211-7
2. Chen S, Yang J, Yang W et al (2020) COVID-19 control in China during mass population movements at New Year. Lancet 395:764-766. https://doi.org/10.1016/s0140-6736(20)30421-9

3. Cyranoski D (2020) What China's coronavirus response can teach the rest of the world. Nature. https://www.nature.com/ articles/d41586-020-00741-x. Accessed 17 Jun 2020

4. Wu JT, Leung K, Leung GM (2020) Nowcasting and forecasting the potential domestic and international spread of the 2019nCoV outbreak originating in Wuhan, China: a modelling study. Lancet 395:689-697. https://doi.org/10.1016/s0140-6736(20) 30260-9

5. News $\mathrm{C}$ Where more than 5 million have people been left Wuhan? https://new.qq.com/omn/20200127/20200127A07OVX00? ADTAG=LenovoPC. Accessed 22 Mar 2020

6. Anderson RM, Heesterbeek H, Klinkenberg D et al (2020) How will country-based mitigation measures influence the course of the COVID-19 epidemic? Lancet 395:931-934. https://doi.org/ 10.1016/s0140-6736(20)30567-5

7. Heymann DL, Shindo N (2020) COVID-19: what is next for public health? Lancet 395:542-545. https://doi.org/10.1016/S01406736(20)30374-3

8. Brooks SK, Webster RK, Smith LE et al (2020) The psychological impact of quarantine and how to reduce it: rapid review of the evidence. Lancet 395:912-920. https://doi.org/10.1016/s01406736(20)30460-8

9. Rubin GJ, Wessely S (2020) The psychological effects of quarantining a city. BMJ 368:m313. https://doi.org/10.1136/bmj.m313

10. Gauthier S, Cummings J, Ballard C et al (2010) Management of behavioral problems in Alzheimer's disease. Int Psychogeriatr 22:346-372. https://doi.org/10.1017/s1041610209991505

11. China NHCotPsRo (2020) No new coronavirus cases in Wuhan sends encouragement to world. http://en.nhc.gov.cn/2020-03/ 20/c_78012.htm. Accessed 20 Mar 2020

12. Barguilla A, Fernández-Lebrero A, Estragués-Gázquez I et al (2020) Effects of COVID-19 pandemic confinement in patients with cognitive impairment. Front Neurol 11:589-590. https://doi. org/10.3389/fneur.2020.589901

13. Lara B, Carnes A, Dakterzada F et al (2020) Neuropsychiatric symptoms and quality of life in Spanish patients with Alzheimer's disease during the COVID-19 lockdown. Eur J Neurol 27:17441747. https://doi.org/10.1111/ene.14339

14. Di Santo SG, Franchini F, Filiputti B et al (2020) The effects of COVID-19 and quarantine measures on the lifestyles and mental health of people over 60 at increased risk of dementia. Front Psychiatry 11:578628. https://doi.org/10.3389/fpsyt.2020.578628

15. Cagnin A, Di Lorenzo R, Marra C et al (2020) Behavioral and psychological effects of coronavirus disease-19 quarantine in patients with dementia. Front Psychiatry 11:578015. https://doi.org/10. 3389/fpsyt.2020.578015

16. Rainero I, Bruni AC, Marra C et al (2020) The impact of COVID19 quarantine on patients with dementia and family caregivers: a nation-wide survey. Front Aging Neurosci 12:625781. https://doi. org/10.3389/fnagi.2020.625781

17. Canevelli M, Valletta M, Blasi MT et al (2020) Facing dementia during the COVID-19 outbreak. J Am Geratr Soc 68:1673-1676. https://doi.org/10.1111/jgs.16644

18. Yang Y, Li W, Zhang Q et al (2020) Mental health services for older adults in China during the COVID-19 outbreak. Lancet Psychiatry 7:e19. https://doi.org/10.1016/s2215-0366(20)30079-1

19. Le Galès C, Bungener M (2016) The family accompaniment of persons with dementia seen through the lens of the capability approach. Dementia 18:55-79. https://doi.org/10.1177/14713 01216657476

20. Arvanitakis Z, Shah RC, Bennett DA (2019) Diagnosis and management of Dementia: review. JAMA 322:1589-1599. https://doi. org/10.1001/jama.2019.4782 
21. Barbe C, Morrone I, Novella JL et al (2016) Predictive factors of rapid cognitive decline in patients with Alzheimer Disease. Dement Geriatr Cogn Dis Extra 6:549-558. https://doi.org/10. 1159/000450975

22. Sadowsky CH, Galvin JE (2012) Guidelines for the management of cognitive and behavioral problems in Dementia. J Am Board Fam Med 25:350-366. https://doi.org/10.3122/jabfm.2012.03. 100183

23. Le S, Zheng' an L, Jianyu Q et al (2020) Prevalence of and risk factors associated with mental health symptoms among the general population in China during the coronavirus disease 2019 pandemic. JAMA Netw Open 3:e2014053. https://doi.org/10.1001/ jamanetworkopen.2020.14053

24. Cuiyan W, Riyu P, Xiaoyang W et al (2020) Immediate psychological responses and associated factors during the initial stage of the 2019 coronavirus disease (COVID-19) epidemic among the general population in China. Int J Environ Res Public Health 17:1729. https://doi.org/10.3390/ijerph17051729

25. Huang Y, Wang Y, Wang H et al (2019) Prevalence of mental disorders in China: a cross-sectional epidemiological study. Lancet Psychiatry 6:211-224. https://doi.org/10.1016/s2215-0366(18) 30511-x

26. Phillips MR, Zhang J, Shi Q et al (2009) Prevalence, treatment, and associated disability of mental disorders in four provinces in China during 2001-05: an epidemiological survey. Lancet 373:2041-2053. https://doi.org/10.1016/s0140-6736(09)60660-7

27. Lu S, Reavley N, Zhou J et al (2018) Depression among the general adult population in Jiangsu Province of China: prevalence, associated factors and impacts. Soc Psychiatry Psychiatr Epidemiol 53:1051-1061. https://doi.org/10.1007/s00127-018-1568-0

28. Wilder-Smith A, Freedman DO (2020) Isolation, quarantine, social distancing and community containment: pivotal role for old-style public health measures in the novel coronavirus (2019nCoV) outbreak. J Travel Med. https://doi.org/10.1093/jtm/taaa0 20

29. Wang H, Xie H, Qu Q et al (2019) The continuum of care for dementia: needs, resources and practice in China. J Glob Health 9:020321. https://doi.org/10.7189/jogh.09.020321

30. Liu S, Yang L, Zhang C et al (2020) Online mental health services in China during the COVID-19 outbreak. Lancet Psychiatry 7:e17-e18. https://doi.org/10.1016/S2215-0366(20)30077-8

31. Kales HC, Gitlin LN, Lyketsos CG (2015) Assessment and management of behavioral and psychological symptoms of dementia. BMJ 350:h369. https://doi.org/10.1136/bmj.h369

32. Volkert D, Chourdakis M, Faxen-Irving G (2015) ESPEN guidelines on nutrition in dementia. Clin Nutr 34:1052-1073. https:// doi.org/10.1016/j.clnu.2015.09.004

33. Volkert D, Beck AM, Cederholm T (2019) ESPEN guideline on clinical nutrition and hydration in geriatrics. Clin Nutr 38:10-47. https://doi.org/10.1016/j.clnu.2018.05.024

Publisher's Note Springer Nature remains neutral with regard to jurisdictional claims in published maps and institutional affiliations. 